\title{
CORRECTIONS
}

\section{Author Correction: Polygenic adaptation: a unifying framework to understand positive selection}

\author{
Neda Barghi@, Joachim Hermisson (1) and Christian Schlötterer(i)
}

Nature Reviews Genetics (2020) https://doi.org/10.1038/s41576-020-0250-z Published online 29 June 2020

The Supplementary information of this article has been updated to correct some errors in the simulation parameters. In the 'fitness function' column the trait optimum for the ' $3 \mathrm{c}-\mathrm{d}$ ' and ' $3 \mathrm{e}$-f' rows has been corrected, and fitness function values are now included for the ' $3 \mathrm{~h}$ ' column. The ' $G$ ' footnote has been updated to indicate that it refers also to figures $3 \mathrm{a}$ and $3 \mathrm{~b}$, not just figure $3 \mathrm{~h}$.

https://doi.org/10.1038/s41576-020-0276-2 I Published online 6 August 2020

(c) Springer Nature Limited 2020

\section{Author Correction: The evolving metabolic landscape of chromatin biology and epigenetics}

Ziwei Dai D, Vijyendra Ramesh (D) and Jason W. Locasale (D)

Nature Reviews Genetics (2020) https://doi.org/10.1038/s41576-020-0270-8 Published online 09 September 2020

Figure 2 of this article has been updated to correct the order of the metabolites succinate and succinyl-CoA (SucCoA) in the tricarboxylic acid (TCA) cycle.

https://doi.org/10.1038/s41576-020-00291-y I Published online 25 September 2020

(๑) Springer Nature Limited 2020 